\title{
Optimism and Female Volleyball Players' Perceptions of Psychological Momentum
}

\author{
Brian Foster \\ Florida State University \\ Selen Razon \\ Ball State University \\ Lindsey C. Blom \\ Ball State University \\ Jocelyn Holden \\ Ball State University
}

\begin{abstract}
Many athletes and spectators believe that experiencing and controlling psychological momentum is a critical component to achieving success in sport (Perreault, Vallerand, Montgomery, \& Provencher, 1998; Stanimirovic \& Hanrahan, 2004). Nevertheless, little is known regarding why some individuals perceive psychological momentum differently than others. This study was designed to determine if optimistic thinking has a relationship with psychological momentum perceptions in sport. Female Division I NCAA volleyball players ( $N=$ 68) completed the Life Orientation Test - Revised (Scheier, Carver, \& Bridges, 1994), the Sport Attributional Style Scale - Short (Hanrahan \& Grove, 1990b), and a psychological momentum assessment. The results indicated that the attributional style constructs of intentionality and globality were significant predictors of psychological momentum perceptions. Also, participants had greater disagreement regarding the momentum value of early and late points in a set than those in between. Future attempts to measure psychological momentum perceptions should consider a mixed methods approach along with more ecologically valid assessment protocols.
\end{abstract}


In sport, athletes and spectators believe that obtaining and controlling psychological momentum (PM) is a critical factor to achieve success (Perreault, Vallerand, Montgomery, \& Provencher, 1998; Stanimirovic \& Hanrahan, 2004). PM is defined as "a positive or negative change in cognition, affect, physiology, and behavior caused by an event or series of events that will result in a commensurate shift in performance and competitive outcome” (Taylor \& Demick, 1994, p. 54). It is closely related to the "hot hand" phenomenon whereby "a streak of previous successes increases an athlete's odds for success on future attempts above the athlete's base rate” (Koehler \& Conley, 2003, p. 253).

Evidence from social psychology suggests that an individual's perception of an event influences their behavior to match that perception. This is termed the perception-behavior link (Dijksterhuis \& Bargh, 2001), and it can occur consciously or unconsciously (Ferguson \& Bargh, 2004). Drawing upon the perception-behavior link, the study of PM can prove beneficial because athletes' perception of PM may influence their subsequent performance (Jones \& Harwood, 2008). The present study sought to examine how minimal exposure to match scores influence perception of PM in athletes. This is important because even minimal exposure to an event can influence affect and perceptions (Zajonc, 1980) and perceptions can impact outcome performance.

There have been three theories regarding the development of PM and its effect on the performance of athletes. According to the AntecedentsConsequences Psychological Momentum Model (Vallerand, Colavecchio, \& Pelletier, 1988) PM is a combination of personal and situational factors. The theory stipulates that the perception of an increased likelihood of goal attainment facilitates a positive change in performance through an increase in motivation and positive emotions. The opposite is true, however, in situations of decreased perceived likelihood of goal attainment. Additionally, based on the model, the degree to which performance is mediated by PM depends on both the context and one's personal beliefs. Given that PM is an exclusively perceptual phenomenon, only the individual's subjective frame of reference impacts PM development. This model of PM was the first to consider that PM is both a cause and an effect. This said, it arguably lacked a detailed explanation of the PM-performance relationship.

A second model of PM is the Projected Performance Model (Cornelius, Silva, Conroy, \& Petersen, 1997). According to the model positive or negative $\mathrm{PM}$ is associated with a shift away from mean performance. Inhibitory or facilitative forces, such as an error or a successful shot, naturally bring performance back to normal levels. PM is considered to be an explanation of performance level after it has occurred (Moesch \& Apitzsch, 2012). This model raised concerns that PM was simply a label of enhanced or depressed performance and in fact not a tangible psychological event. To test such concerns various 
research attempts were made to assess PM from a statistical perspective known as the hot hand phenomenon. Gilovich, Vallone, and Tversky (1985) were the first to dispute conventional sports wisdom when they analyzed data from the Philadelphia 76ers' 1980-1981 season and found no evidence of shooting streaks that would be greater than expected by chance. Subsequent studies found similar results in sports such as golf (Clark, 2004), volleyball (Miller \& Weinberg, 1991), and tennis (Silva, Hardy, \& Crace, 1988). There have been studies, however, that have identified hot hand in billiards (Adams, 1995) and bowling (DorseyPalmateer \& Smith, 2004). Also, athletes have self-reported that they feel the influence of PM while they play (Jones \& Harwood, 2008). Hales (1999) had also initially proposed that the relationships among PM, hot hand phenomenon, and performance should be studied together. Therefore the model could be criticized for not considering athletes' subjective frame of reference during sport performance.

A third model of PM is the Multidimensional Model of Momentum (Taylor \& Demick, 1994). This model posits that PM is the result of a chain of events. Specifically, PM is a five-step process: 1) a precipitating event; 2) a change in cognition, affect, and physiology; 3) a change in behavior; 4) an increase or decrease in performance; and 5) an immediate change in outcome. A strength of the model is that it emphasizes subjective interpretation of an event. This is important because Perreault et al. (1998) have shown that a negative precipitating event does not necessarily have to hinder performance.

$\mathrm{PM}$ research has been equivocal in nature. A major reason for this is that two individuals can perceive the PM of a sport event very differently (Burke, Aoyagi, Joyner, \& Burke, 2003). There is currently limited research on the underlying mechanisms that influence PM perceptions (Alter \& Oppenheimer, 2006). As a result, there is a need for research that identifies the psychological variables that may affect individuals' subjective perception of PM.

One such variable that could possibly cause individuals to perceive PM differently is optimism. Positive thought processes have been demonstrated to enhance performance (Tod, Hardy, \& Oliver, 2011). These thought processes change cognition and affect and are therefore critical antecedents of PM per the Multidimensional Model of Momentum.

Optimism can be manifested as dispositional optimism, where optimism is a stable disposition and the individual has subjective beliefs that future outcomes are bound to be positive (Scheier \& Carver, 1985). Alternatively, optimism can be manifested as an attributional style, which is termed as the manner in which individuals explain their successes and failures to themselves or others (Alloy, Peterson, Abramson, \& Seligman, 1984). As such, optimistic individuals prefer stable, global, and internal explanations for their successes, and unstable, specific, and external explanations for their failures (Seligman, Nolen-Hoeksema, 
Thornton, \& Thornton, 1990). In sport environments, optimism has been shown to be a predictor of future success because it is correlated with positive affect (Sanjuán, Pérez, Rueda, \& Ruiz, 2008), psychological resilience (Davis \& Asliturk, 2011), and proactive coping (Aspinwall, Sechrist, \& Jones, 2005; Sohl \& Moyer, 2009). Additionally, optimistic individuals have been shown to achieve better psychological adjustment during sport participation (Armata \& Baldwin, 2008).

Consistent with previous assertions, research into the common qualities of high achieving Olympic athletes revealed twelve common characteristics among which optimism and psychological resilience were two (Gould, Dieffenbach, \& Moffett, 2002). Optimistic individuals tend to view adversity as a challenge that can be overcome with effort and persistence (Schulman, 1999). Research has indicated a link between optimistic thinking and improved performance outcomes in swimming (Seligman et al., 1990). Specifically, at competitive meets those swimmers with pessimistic explanatory style were most likely to perform below expectations throughout the season. Also, relative to swimmers with more optimistic styles, pessimistic swimmers' subsequent performances were more negatively affected following a poor meet.

The purpose of this study was to assess the relationship between PM perceptions and optimism in collegiate female volleyball players. It was hypothesized that both dispositional optimism and optimistic attributional style would have positive relationships with perceptions of PM. In addition, it was hypothesized that dimensions constituting optimistic attributional style (i.e., internality, stability, globality, controllability, and intentionality) would be significant predictors of PM perceptions. Establishing a relationship between optimism and PM perceptions could have applied ramifications for designing performance-enhancement interventions targeting optimism in athletes because experiencing PM has been long demonstrated to improve performance (Perreault et al., 1998).

\section{Participants}

\section{Methods}

A purposeful sample of 68 female NCAA Division I volleyball players from 17 athletic conferences were recruited for the study. Specifically the purposeful sample was geared towards maximizing the number of participants within one sport category, in season at the time when the data was collected. Only female players were tested because there were substantially more female NCAA Division I volleyball teams than male teams to send the questionnaires to. Participants were 18-21 years of age with a mean age of 19.53 years. Participants' mean years of competitive volleyball experience was 7.87 years. To recruit the participants for the study, Division I volleyball coaches were initially contacted 
and asked to forward the study's link to their players. The link included the informed consent for participation as well as the questionnaires for the study. A repeat email was sent to the same coaches two weeks following this initial attempt. Finally, to best maximize the sample size, three weeks following the second attempt, 200 assistant coaches were personally emailed by the researcher. Prior to any data collection, approval to conduct this study was obtained from the researchers' university Institutional Review Board (IRB).

\section{Instruments}

Participants were asked to complete a demographic questionnaire, the Life Orientation Test - Revised (Scheier, Carver, \& Bridges, 1994), the Sport Attributional Style Scale - Short (Hanrahan \& Grove, 1990b), and the Psychological Momentum Assessment (PMA).

Demographics Questionnaire. The questionnaire included items gauging participants' age, class, race, athletic conference, and years of competitive volleyball experience.

Life Orientation Test - Revised (LOT-R; Scheier et al., 1994). LOT-R measured dispositional optimism. The items included ten statements rated from 0 (strongly disagree) to 4 (strongly agree). Example statements were "in uncertain times, I usually expect the best", and "I'm always optimistic about my future". A final dispositional optimism score of 0-24 corresponded to the summations of ratings from six of the statements that measured dispositional optimism. The correlation between the original LOT scale and the LOT-R is .95. LOT-R possesses a stronger focus on positive expectations (Scheier et al., 1994), hence its use for the purposes of the study. For the current study, a Cronbach's alpha coefficient of .823 was computed for the six items of the scale measuring dispositional optimism.

\section{Sport Attributional Style Scale - Short. (SASS-S; Hanrahan \& Grove,} 1990b). The scale measured sport-related attributional style along the five dimensions of internality, stability, globality, controllability, and intentionality for both positive and negative events" (Hanrahan \& Grove, 1990a, p. 183). Of the ten items, five included positive events and five included negative events. An example positive item was "your teammates claim that you are a very good volleyball player". An example negative item was "you are not selected for the starting line-up in an important match". For each item of the scale, participants identified the single most likely cause of that event and responded to five prompts, each corresponding to one of the five dimensions of attributional style. The total score for the scale ranged 10-70. Higher scores indicated that the participant attributed the cause of the events to internal, stable, global, uncontrollable, and unintentional factors. To compute an optimistic attributional style score, the total dimension scores for internality, stability, and globality were 
summed. The optimistic attributional style score ranged 30-210, with a higher score representing a greater optimistic attributional style.

SASS-S is valid and correlated with the original SASS at $r=.92$ (Hanrahan \& Grove, 1990b). The instrument has good construct validity (Hanrahan, Grove, \& Hattie, 1989) and was used in similar investigations to measure optimism in athletes (Parkes \& Mallett, 2011). For the purposes of the current study, the SASS-S was slightly modified to increase fit to volleyball. For example, Item 6 was changed from "your teammates claim that you are not a good performer" to "your teammates claim that you are not a good volleyball player". It should also be noted that the original SASS-S included seven questions for each item. In the present study, the questions specific to importance and image clarity were eliminated because they were not of interest.

Psychological Momentum Assessment (PMA). The PMA assessed PM perceptions. The scale was adapted from a previous PM scale used to identify tennis players' perceived match momentum following each game (Vallerand et al., 1988). Using this scale, participants rated the momentum from 1 (Player $A$ definitely has the momentum) to 7 (Player B definitely has the momentum) with a neutral midpoint of 4 (neither player has the momentum). For the purposes of this study, participants used the PMA to rate the momentum following each point of the fifth set of a hypothetical championship volleyball match where they imagined themselves as a player on Team " $A$ ".

Specifically herein, the score of the match was presented to the participant after each point. After viewing which team had scored participants were asked to rate the current PM of the match from 1 (Team A definitely has the momentum) to 7 (Team $B$ definitely has the momentum) with a neutral midpoint of 4 . The final score of the set was 15-13 for Team B. The pattern of scores was presented in manner that the largest disparity in score at any time was 8-5 for Team B. Neither team scored more than two consecutive points in the set. Each participant's PM score was derived from the sum of all scores provided. Means and standard deviations were also analyzed for each point.

Both in tennis and volleyball, analyzing the dynamics of the game per the players' mind involve similar processes (Notarnicola et al., 2014) hence the effective adjustment of the original scale to the purposes of the present study. Also, in line with the notion of immediate retrospective verbal recall (North, Ward, Ericsson, \& Williams, 2011), in psychology research, hypothetical scenarios are commonly used (Dubuc, Schinke, Eys, Battochio, \& Zaichkowsky 2010; FeldmanHall et al., 2012) to prompt thoughts and attitudes, thereby the use of a hypothetical approach in the present framework. 


\section{Procedure}

The study included one online session of survey administration via the Qualtrics online survey system. Upon proceeding to the link for the study, participants viewed and signed the informed consent from. Subsequently, participants completed the demographics form. Finally, the LOT-R, SASS-S, and PMA were administered in random order. Counterbalancing the administration order of the instruments was necessary in order to control for testing effects. The mean data collection time per participant was approximately 15 minutes.

\section{Research Design and Analysis}

The study used a correlational design. Descriptive statistics were run for mean age and competitive volleyball experience of the participants, as well as means for the LOT-R, SASS-S, and PMA. For the first hypothesis a Pearson's correlation was run between LOT-R total scores and PM total scores to determine if dispositional optimism had a significantly positive relationship with PM perceptions. The second hypothesis was tested using a Pearson's correlation between optimistic attributional style scores (i.e., sum of internality, stability, and globality subscales) and PM total scores to determine if there was a significantly positive relationship between optimistic attributional style and PM perceptions. The third hypothesis was tested with a standard multiple linear regression run on PM scores and the five SASS-S subscales. All alpha levels were established at .05 , and statistical analyses were run using the Statistical Package for the Social Sciences (SPSS) 20.0 software.

\section{Results}

Of the 176 participants who initially signed up, 68 completed all the measures. Therefore the analyses were run on the data provided by these 68 participants. Descriptive statistics were run to determine means and standard deviations for age, competitive volleyball experience, dispositional optimism score, sport-related optimistic attributional style and its five subscales, and PM perceptions (see Table 1). The mean age of the participants was 19.53 years and their mean competitive volleyball experience was 7.87 years. Caucasians represented $80.6 \%$ of the sample, African Americans or blacks $7.5 \%$, and Hispanics 5.9\%. In addition to the final score derived from the PMA, descriptive statistics were run for each point of the PMA. 
Table 1

Participant Age, Experience, LOT-R, SASS-S, Internality, Stability, Globality, Controllability, Intentionality, and PM Means, Standard Deviations, and Range

\begin{tabular}{lccc}
\hline & Mean & $\begin{array}{c}\text { Standard } \\
\text { Deviation }\end{array}$ & Range \\
\hline Age & 19.53 & 1.17 & $18-21$ \\
Experience & 7.87 & 2.79 & $2-18$ \\
LOT-R & 16.19 & 4.23 & $2-23$ \\
SASS-S & 128.00 & 12.39 & $98-163$ \\
Internality & 40.44 & 5.19 & $27-52$ \\
Stability & 44.74 & 5.50 & $34-62$ \\
Globality & 42.62 & 5.93 & $23-60$ \\
Controllability & 37.60 & 6.00 & $18-49$ \\
Intentionality & 29.43 & 9.36 & $10-47$ \\
PM & 115.64 & 10.98 & $57-142$ \\
\hline
\end{tabular}

Note: LOT-R possible range 0-24, SASS-S 30-210, Internality, Stability, Globality, Controllability, and Intentionality all 10-70, PM 27-189

In regard to the first research question addressing the relationship between dispositional optimism and PM perceptions, no correlation was found, $r=.000, p$ $=.999$. Thus descriptively speaking, the results indicated no relationship between dispositional optimism and PM perceptions.

For the second research question addressing optimistic attributional style and PM perceptions, no correlation was found, $r=-.020, p=.872$. Thus, results indicated no correlation between sport-specific optimistic attributional style and PM perceptions. Among subscales, internality and stability revealed a nonsignificant, weak, positive correlation, $r=.223, p=.068$, internality and globality revealed a significant, weak to moderate, positive correlation, $r=.341, p=.004$, and stability and globality revealed a significant, moderate, positive correlation, $r$ $=.553, p<.001$.

A Pearson's correlation was also run between dispositional optimism and sport-specific optimistic attributional style as measured by the LOT-R and SASS$\mathrm{S}$ respectively. A significant, weak to moderate, positive correlation was found, $r$ $=.300, p=.013$. Thus, individuals with higher dispositional optimism also scored higher on sport-specific attributional style. 
Finally, for the third research question addressing attributional style subscales and PM perceptions, a standard multiple linear regression was run with scores from the five SASS-S subscales and PMA scores (see Table 2). Among the five SPSS-S subscales, intentionality, $b=.315, p=.048$, and globality, $b=-.678$, $p=.019$, were found to significantly predict PM perceptions. Thus, the stronger the belief an athlete had that sport successes are caused by intentional action, the more likely they were to perceive PM in their favor. Also, the stronger the belief an athlete had that the cause of a sport-related success had only sport-specific relevance, the more likely they were to perceive PM in their favor. However, altogether the five SASS-S subscales did not explain a significant proportion of variance in PM perceptions, $R^{2}=.166, F(5,63)=2.31, p=.055$.

Table 2

Simple Linear Regression of SASS-S Subscales and PM Scores

\begin{tabular}{lccc}
\hline \multicolumn{1}{c}{ Variable } & $\begin{array}{c}\text { Unstandardized } \\
\text { Beta Coefficients }\end{array}$ & $\begin{array}{c}\text { Standardized Beta } \\
\text { Coefficients }\end{array}$ & Significance \\
\hline (Constant) & 128.261 & & .000 \\
Internality & .047 & .023 & .861 \\
Stability & .577 & .269 & .059 \\
Globality & -.678 & -.350 & .019 \\
Controllability & .057 & .031 & .814 \\
Intentionality & .315 &, 268 & .048 \\
$R^{2}=.166, F(5,63)=2.31, p=.055$ & &
\end{tabular}

Post-hoc findings to consider herein include some of the descriptive data associated with the PMA (see Figure 1). Each participant rated the current PM of the match after each point. Noteworthy is that the largest standard deviation value corresponded to the first point. Essentially, some participants were convinced that winning the first point of the set was critically important, whereas other participants deemed the point to have little effect on PM. In addition, the standard deviations of the PM ratings of the last five points were higher than most points that came earlier. These findings suggest that there may be less agreement among volleyball players regarding the PM value of early and late points in a set or match. 
Figure 1. Psychological Momentum assessment data displaying participants' mean PM ratings for each point and standard deviations of those ratings.

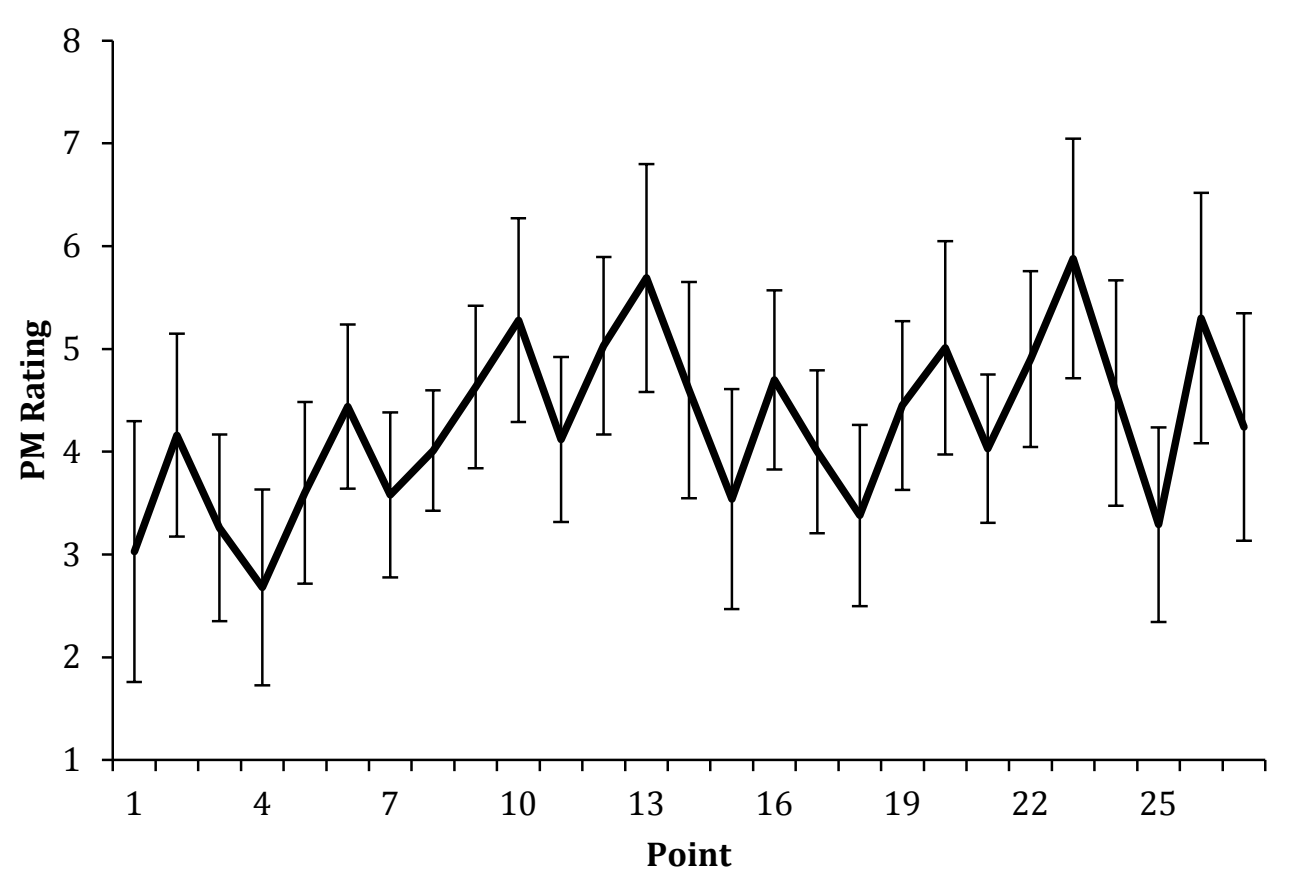

\section{Discussion}

The purpose of this study was to determine if optimistic thinking is associated with PM perceptions. Attributional style constructs intentionality and globality were found to be significant predictors of PM perceptions. Additionally, it was found that volleyball players tend to have greater disagreement regarding the PM value of points early and late in a set than those points in between.

For the first research question it was hypothesized that dispositional optimism would be related to PM perceptions. The results did not support this hypothesis.

Dispositional optimism did not correlate with PM perceptions. In an effort to explain the unexpected results, a reexamination of PM theory and the PMA is necessary. Specifically, the Antecedents-Consequences Psychological Momentum Model (Vallerand et al., 1988) proposes that PM is a product of context and personal beliefs. As such, the model purports that emotions and feelings of control, confidence, motivation, and energy precede PM perceptions in individuals. 
Providing that contextual cues (i.e. spectators, officials, and playing conditions) were not inherent in the present study, it may well be that the PMA herein failed to elicit the emotions and feelings that are otherwise necessary for true PM perceptions to occur. Further adding to these perspectives, in their Multidimensional Model of Momentum Taylor and Demick (1994) conceptualized PM as unfolding in a five-step sequence: 1) a precipitating event; 2) a change in cognition, affect, and physiology; 3) a change in behavior; 4) an increase or decrease in performance; and 5) an immediate change in outcome. Arguably due to the written nature of the present assessment format, the participants in this study may not have fully experienced this process. Similar to the importance of affect to PM perceptions, the importance of positive affect associated with dispositional optimism has also been stressed (Sanjuán et al., 2008). Altogether, a possible explanation for the lack of significant findings may still lie in the lack of affect derived in participants during the study. In fact, from a methodological standpoint, it is well known that surveys and assessments may present ecological shortcomings in regards to eliciting and measuring psychological states (Cicourel, 2007).

Alternatively, dispositional optimism and PM perceptions may not be related constructs. Kerick et al. (2000) have for instance suggested that the role of affect and physiology on perceptions of PM may be overstated. PM perceptions may in fact have more to do with sport-specific knowledge than any dispositional quality.

For the second research question the findings revealed no correlation between sport-specific optimistic attributional style and PM perceptions. While research has shown that more optimistic individuals prefer stable, global, and internal explanations for their successes, and unstable, specific, and external explanations for their failures (Seligman, 1990), these findings were not replicated in the current study. It may be that the participants in this study did not take ownership of "winning" or "losing" despite the instructions to do so. More recently, Gernignon, Briki, and Eykens (2010) stressed that PM perceptions were more likely to develop when an athlete is pursuing a goal he/she perceives as important. No measurement in the current study was used to assess the inherent value that participants placed upon the goal of "winning" in the experimental script. The current study's general problem in ecological validity may have been remedied by asking the respondents to image the particular scenario in order to best elicit the emotional responses preceding PM perceptions. Alternatively, a more natural and ecologically valid environment could have been simulated by using props, showing a previously recorded game, or administering commitment and manipulation checks to gauge participants' imagery ability before and during the assessment. 
For the third research question, a simple linear regression with the SASS$\mathrm{S}$ subscales PM scores revealed that intentionality and globality were significant predictors of PM perceptions. Intentionality was determined to be a significant predictor of PM perceptions, $b=.315, p=.048$. This finding suggests that to the extent that an individual associates intentional action with sport success, they will perceive PM to be in their favor. This finding is consistent with previous literature that indicates that individuals believe PM and hot streaks may be contingent upon one's intentional actions (Roney \& Trick, 2009). Essentially, PM is thought to be a quality that can be forcefully developed with the correct skills and strategy. Globality was also found to be a significant predictor of PM perceptions, $b=$ $.678, p=.019$. This finding suggests that the stronger the belief an athlete has that the cause of a sport-related success has only sport-specific relevance, the more likely they are to perceive PM in their favor. A possible explanation for this finding could be that competitive female athletes tend to make fewer global attributions than recreational female athletes (Hanrahan \& Cerin, 2009). Through experience competitive athletes may develop an understanding that their athletic self-concept is separate from their self-concept in other aspects of life.

In contrast to intentionality and globality, the subscales of stability, controllability, and internality were determined not to be significant predictors of PM perceptions. Stability was close to being a significant predictor, $b=.577, p=$ .059 . This is understandable given the fact that globality and stability are highly correlated with one another. A relatively surprising finding was that controllability was not a significant predictor of PM perceptions, $b=.057, p=$ .814. Controllability has been argued to be similar to intentionality (Russell, 1982; Weiner, 1985). The inconsistency of this finding may be attributable to the lack of a natural sport environment while measuring PM. The study design may not have elicited feelings of control to the extent necessary to influence PM perceptions. Consequently from a methodological perspective, future studies ought to investigate the ecological validity of the PM instruments used herein. Given that PM is among the most elusive concepts to capture (Moesch \& Apitzch, 2012), improvement of its measurement tools would benefit the field. Finally, internality was also not found to be a significant predictor of PM perceptions either, $b=$ $.047, p=.861$. This finding is not as surprising given that previous research has shown that internality has very little impact on expectations, which in turn may mediate PM perceptions (Peterson \& Vaidya, 2001).

Beyond the previously discussed research questions, post-hoc analyses revealed moments of particular interest where there was greater discrepancy among participants with their PM ratings. Noteworthy is that the largest standard deviation value corresponded to the first point. Essentially, some participants were convinced that winning the first point of the set was critically important, whereas other participants deemed the point to have little effect on PM. Previous 
research has also indicated some dissension among observers regarding momentum points in basketball (Burke et al., 2003) and tennis (Burke, Edwards, Weigand, \& Weinberg, 1997). Future studies should address this observation and determine if this is indeed the case.

While some of the findings of this study were not as expected, they may still lead to practical implications. Intentionality was for instance found to be a significant predictor of PM perceptions. To the extent that an individual associates intentional action with sport success, they will perceive PM to be in their favor. Practitioners may then educate athletes that cognitions, affect, behaviors, and perceived goal progression are factors that can be optimized through intentional effort. That is, athletes may get to perceive that they aren't merely at the whim of their environment because they are capable of changing their cognitions, affect, and behaviors through their own will for their own benefit. Second, PM perceptions at times can be unclear and all athletes may not perceive PM similarly. Traditional sport psychology consulting wisdom emphasizes maintaining focus only on the point at hand and to value each point similarly (Mack \& Casstevens, 2001). Sport psychology consultants may benefit from emphasizing this point-by-point awareness especially to render PM perceptions more clear to the athletes.

For all of the findings presented herein, the lack of ecological validity has been highlighted as an important shortcoming. Two additional limitations must be considered. First, the sample size was smaller than desired for correlation and regression research and may have contributed to the lack of significant findings. Second, the sample was sport-specific and gender-specific, which limits the generalization of present observations across alternative sports and populations.

To reiterate an important point from the previous discussion, a major obstacle that has prevented PM from being studied more effectively is that it remains a difficult concept to capture quantitatively due to its abstract nature (Moesch \& Apitzch, 2012). Therefore, future research is warranted to develop more ecologically valid measurement protocols for best capturing PM. To that end, adding a qualitative perspective to the PM measurement framework may prove beneficial (Crust \& Nesti, 2006). Consequently, practical implications drawn from more in-depth findings may help shape psychological skills training programs to promote positive PM perceptions in athletes. 


\section{References}

Adams, R. M. (1995). Momentum in the performance of professional tournament pocket billiards players. International Journal of Sport Psychology, 26(4), 580-587.

Alloy, L. B., Peterson, C., Abramson, L. Y., \& Seligman, M. E. P. (1984). Attributional style and the generality of learned helplessness. Journal of Personality and Social Psychology, 46(3), 681-687.

Alter, A. L., \& Oppenheimer, D. M. (2006). From a fixation on sports to an exploration of mechanism: The past, present, and future of hot hand research. Thinking \& Reasoning, 12(4), 431-444.

Armata, P. M., \& Baldwin, D. R. (2008). Stress, optimism, resiliency, and cortisol with relation to digestive symptoms or diagnosis. Individual Differences Research, 6(2), 123-138.

Aspinwall, L. G., Sechrist, G. B., \& Jones, P. R. (2005). Expect the best and prepare for the worst: Anticipatory coping and preparations for Y2K. Motivation and Emotion, 29, 357-388.

Burke, K. L., Aoyagi, M. W., Joyner, A. B., \& Burke, M. M. (2003). Spectators' perceptions of positive momentum while attending NCAA men's and women's basketball regular season contests: Exploring the antecedentsconsequences model. Athletic Insight, 5(3), 10-18.

Burke, K. L., Edwards, T. C., Weigand, D. A., \& Weinberg, R. S. (1997). Momentum in sport: A real of illusionary phenomenon for spectators. International Journal of Sport Psychology, 28, 79-96.

Cicourel, A. V. (2007). A personal, retrospective view of ecological validity. Text \& Talk, 27(5/6), 735-752.

Clark, R. D. (2004). On the independence of golf scores for professional golfers. Perceptual \& Motor Skills, 98(2), 675-681.

Cornelius, A., Silva III, J.M., Conroy, D. E., \& Petersen, G. (1997). The projected performance model: Relating cognitive and performance antecedents of psychological momentum. Perceptual \& Motor Skills, 84(2), 475-485.

Crust, L., \& Nesti, M. (2006). A review of psychological momentum in sports: Why qualitative research is needed. Athletic Insight, 8(1), 1-15. Retrieved from http://www. athleticinsight.com/Vol8Iss1/MomentumPDF.pdf.

Davis, C. G., \& Asliturk, E. (2011). Toward a positive psychology of coping with anticipated events. Canadian Psychology, 52(2), 101-110.

Dijksterhuis, A., \& Bargh, J. A. (2001). The perception-behavior expressway: Automatic effects of social perception on social behavior. Advances in Experimental Social Psychology, 33, 1-40.

Dorsey-Palmateer, R., \& Smith, G. (2004). Bowlers' hot hands. American Statistician, 58(1), 38-45. 